\title{
Allergy to grass pollen: mapping of Dactylis glomerata and Phleum pratense allergens for dogs by two-dimensional immunoblotting
}

\author{
Luís Miguel Lourenço Martins ${ }^{1,2}$, Andreia Grilo Marques ${ }^{1}$, Luísa Maria Dotti Silva Pereira ${ }^{2,3}$, Saul José Semião-Santos ${ }^{4}$, \\ Ofélia Pereira Bento ${ }^{2,5}$

\begin{abstract}
${ }^{1}$ Department of Veterinary Medicine, School of Sciences and Technology, University of Évora, Évora, Portugal ${ }^{2}$ Institute of Mediterranean Agricultural and Environmental Science (ICAAM), University of Évora, Évora, Portugal ${ }^{3}$ Escola Superior Agrária de Elvas, Polytechnique Institute of Portalegre, Elvas, Portugal ${ }^{4}$ Department of Nursing, GEE, Universidade Tiradentes, Aracaju, Brazil

${ }^{5}$ Department of Animal Science, School of Sciences and Technology, University of Évora, Évora, Portugal
\end{abstract}

Adv Dermatol Allergol 2017; XXXIV (1): 60-69

DOI: https://doi.org/10.5114/ada.2017.65623

\begin{abstract}
Introduction: Much less is known about grass-pollen allergens to dogs, when compared with humans. Geneticbased patterns might play an important role in sensitization profiles, conditioning the success of allergen-specific immunotherapy.

Aim: Mapping of Dactylis glomerata (D. glomerata) and Phleum pratense ( $P$. pratense) allergens for grass pollensensitized atopic dogs, for better understanding how individual allergograms may influence the response to grasspollen immunotherapy.

Material and methods: To identify $D$. glomerata and $P$. pratense allergoms for dogs, 15 individuals allergic to grass pollen and sensitized to $D$. glomerata and P. pratense were selected. D. glomerata and P. pratense proteomes were separated by isoelectric focusing (IEF), one-dimensional (1-D) and two-dimensional (2-D) sodium dodecyl sulphate polyacrylamide gel electrophoresis (SDS-PAGE). Separated proteins were blotted onto Polyvinylidene difluoride (PVDF) membranes and allergens were identified by patient sera IgE in Western Blotting (WB).

Results: In D. glomerata, 17 allergens were identified from IEF and 11 from 1-D SDS-PAGE, while from P. pratense, 18 and 6 allergens were identified, respectively. From 2-D SDS-PAGE 13 spots were identified from D. glomerata and 27 from $P$. pratense.

Conclusions: Several similarities were found between dog and human D. glomerata and P. pratense sensitization profiles but no relationship between clinical signs and a specific pattern of allergen recognition was observed. Similarities were found in each patient pattern of sensitization between $D$. glomerata and $P$. pratense, also suggesting cross-reactive phenomena. Further molecular epidemiology approach is needed to understand the role of the sensitization pattern in allergen-specific immunotherapy effectiveness in grass-pollen allergic dogs.
\end{abstract}

Key words: allergens, atopy, dog, grass pollen allergens, immunoblotting.

\section{Introduction}

After a clinical diagnosis of atopy, which criteria have been successively improved [1], extensive laboratory data are very useful to understand sensitization patterns and for a better clinical follow-up [2]. Intradermal skin testing (IDT) and specific IgE determinations are also useful to establish eviction measures and consider allergenspecific immunotherapy (ASIT), in order to obtain clinical improvement [3]. In fact, allergen-specific immunothera- py, especially for aeroallergens, has proven to be useful for allergy control, by promoting substantial clinical improvement in $65-75 \%$ of the cases [4] and should be prescribed as directed as possible [3, 5]. Nevertheless, those figures may be safely improved by using more specific allergen pools for specific immunotherapy, which is already being studied for human allergy management [6-10].

Studies performed by Kubota et al. [11] with Cryptomeria japonica pollen allergens, using sera from sen-

Address for correspondence: Luís Miguel Lourenço Martins, Department of Veterinary Medicine, School of Sciences and Technology, University of Évora, Núcleo da Mitra, 7000-093 Évora, Portugal, phone: +351 919498570, fax: +351 266760944, e-mail: Imlm@uevora.pt Received: 12.10.2015, accepted: 25.12.2015. 
sitized dogs with atopic dermatitis, showed important differences in terms of individual allergen recognition between humans and dogs. In fact, a molecule that has shown to be a major allergen for humans may be a minor one for dogs and vice versa. Sometimes, an allergen for a given species may even not be so for another. More recently, Ognjenovic et al. [12] reported several similarities, as well as differences, between dog and human sensitization to the Ambrosia artemisiifolia pollen, with 81\% recognition of the $38 \mathrm{kDa}$ band, belonging to the Amb a 1 group, between sensitized dogs.

For a precise allergy diagnosis, or indication of allergen-directed eviction measures [2], as well as to improve the efficacy of allergen-specific immunotherapy [13], further fine tune laboratory information on the molecular diagnosis seems to be truly helpful.

Following very useful concepts, such as componentresolved diagnosis and immunotherapy (CRD and CRIT) [14-16], characterization of patient serum IgE spectrotypes (individual allergograms) by WB techniques, with electrophoretic-separated molecular allergens, may provide relevant information, especially for an immunotherapy decision [13].

For the determination of dog-specific serum IgE, ELISA has been the routine laboratory method, but when comparing with the latest-generation methods, sensitivity and specificity has shown to be lower [17]. However, the lack of standardization of those new methods for veterinary proposal is still an obstacle. An extraordinary advance for allergy complementary diagnosis is based on the protein microarray systems [15]. The Immune Solid-phase Allergen Chip method (ISAC - Thermo Fisher Scientific, USA), despite its solely qualitative and semiquantitative ability, allows the detection of very small amounts of specific lgE, which may nevertheless be of clinical significance [8]. While protein microarray systems are still far from veterinary routine, the characterization of patient IgE sensitization profile by WB over electrophoretic-separated allergens may provide relevant information, especially to understand particular conditions related to cross-reaction phenomena and immunotherapy effectiveness [2, 8, 13].

In this work the authors intended to further map Dactylis glomerata (D. glomerata) and Phleum pratense (P. pratense), two well-known allergen sources for humans [18, 19], allergens for grass pollen-sensitized atopic dogs, in order to settle the necessary conditions to start understanding how individual allergograms may influence the response to ASIT.

\section{Aim}

By mapping D. glomerata and P. pratense allergens for grass pollen-sensitized atopic dogs, the authors intended to disclose the allergens from those two relevant sources for dogs, for further understanding how individual aller- gograms may influence the response to grass-pollen immunotherapy.

\section{Material and methods}

\section{Patient selection and characterization}

At the dermatology and allergy outpatient consultation from the University of Évora Veterinary Hospital (Évora, Portugal), all the patients were submitted to an extensive query for anamnestic and clinical parameters and selected by filling the clinical criteria defined by the International Task Force on Canine Atopic Dermatitis for atopy diagnosis [1]. For further selection, patients were submitted to IDT with different allergen sources, such as grass, herbs and tree pollens, mites and fungi, and positive (histamine solution) and negative (solvent extract) controls from Bial Aristegui (Bilbao, Spain). Specific IgE for a panel of the most common mites and pollens was also determined according to Lee et al. [20] (Univet, Barcelona, Spain). Fourteen of those, mostly indoor patients and presenting atopic dermatitis, which tested positive in the IDT and presented with specific IgE for D. glomerata and $P$. pratense, were selected. Only one patient (no. 6) was already undergoing specific immunotherapy.

\section{Allergen extract preparation}

Lyophilized pollens from D. glomerata and P. pratense (Allergon, Angelholm, Sweden) were submitted to extraction by $2 \mathrm{~h}$ orbital stirring at $1: 10$ in $4^{\circ} \mathrm{C}$ double-distilled water, for IEF, and in phosphate buffered saline (PBS) for SDS-PAGE. The extract was centrifuged at $3500 \mathrm{~g}$ for 10 min at $4^{\circ} \mathrm{C}$ and supernatant protein concentration measured according to the Warburg Christian Method [21] in a spectrophotometer (Beckman DU530 UV-VIS, Beckman Coulter, Brea, USA).

\section{Protein separation}

\section{Isoelectric focusing (IEF)}

Dactylis glomerata and P. pratense extracts (protein concentration of $4.6 \mathrm{mg} / \mathrm{ml}$ ) were subjected to $\mathrm{pH}$ 3-10 IEF (Ampholytes from GE Healthcare, USA) in 5\% polyacrylamide gel (Acrylamide/Bisacrylamide from Bio-Rad, Hercules, USA). Separated proteins were then blotted onto methanol-activated 0.2- $\mu \mathrm{m}$-pore PVDF membranes (Bio-Rad) according to the technique described by Martins et al. [22] for isoelectric-focused protein transference. Both extracts were also subjected to IEF, to produce 1-D gel strips for the 2-D gel electrophoresis.

\section{SDS-PAGE}

Dactylis glomerata and $P$. pratense extracts were treated with SDS, under non-reducing conditions, and submitted to $12 \%$ PAGE. Separated proteins were blotted under electrotransference [23] onto $0.2 \mu \mathrm{m}$-pore PVDF membranes in a Trans-Blot Semi-Dry Transfer Cell 


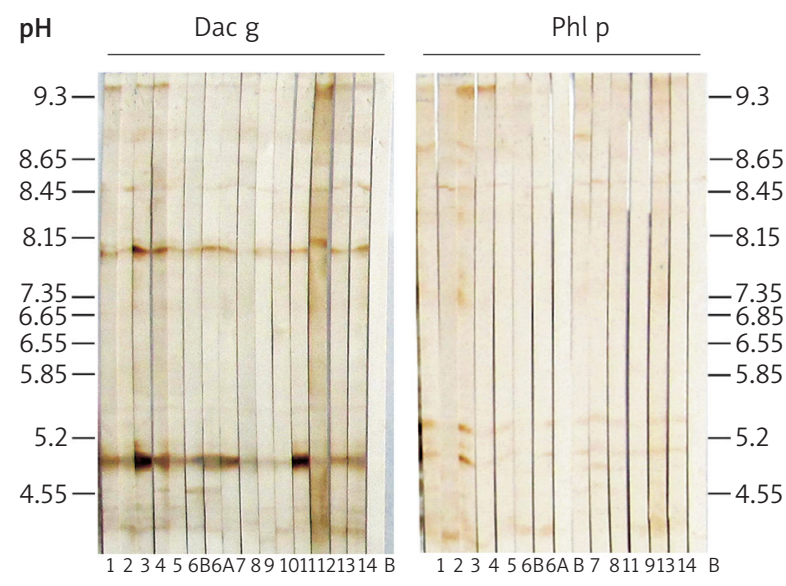

Figure 1. IgE immunoblots from D. glomerata and P. pratense $\mathrm{IEF}$, revealed with horseradish peroxidase-conjugated goat anti-dog-lgE. Patients/lanes no. 1-14. Patient no. 6 before (6B) and after (6A) specific immunotherapy B - Blank controls without serum

(Bio-Rad). Two-dimensional separations were performed by $12 \%$ SDS-PAGE of the 1-D IEF strips and separated proteins were also blotted onto methanol-activated $0.2 \mu \mathrm{m}$ pore PVDF membranes.

\section{Antigen detection - immunoblotting}

One-dimensional blotted membranes were cut in 3 -mm-wide strips and immersed for $2 \times 0.5 \mathrm{~h}$ in blocking buffer (1\% non-fat dry milk - 0.1\% Tween-20 in Trisbuffered saline, at pH 7.4) at room temperature and each strip was incubated overnight with individual patient sera, diluted at 1 : 10 in $1 \mathrm{ml}$ blocking buffer. Fourteen patients' (no. 1 to 14) sera were tested with D. glomerata and $P$. pratense strips, from IEF and from SDS-PAGE. Two samples were used from patient no. 6: one before immunotherapy (6B) and another after a year of immunotherapy (6A). For specific IgE detection, strips were incubated for $2 \mathrm{~h}$ with horseradish peroxidase-conjugated goat anti-dog-IgE antibody (Bethyl Laboratories, Inc., Montgomery, USA) at $1: 1000$ in blocking buffer, followed by $4 \times 5$ min washes in $0.9 \% \mathrm{NaCl}-0.1 \%$ Tween-20. Another $4 \times 5$ min washing cycle was carried out and specific IgE visualization performed by chromogenic reaction with 3.3'-diaminobenzidine $/ \mathrm{NiCl}_{2}$ (Sigma-Aldrich, St. Louis, USA) in Tris-buffered saline at pH 7.4.

Two-dimensional immunoblottings were performed by incubating blotted membranes with a pool of 7-patient sera (no. 1, 2, 3, 5, 6A, 13 and 14), selected for their representative IgE binding in IEF and 1-D SDS-PAGE blots, diluted to $1: 10$ in $18 \mathrm{ml}$ blocking buffer. Specific IgE was detected by anti-dog-IgE biotin-labeled monoclonal antibody (AbD Serotec, Kidlington, UK) at $1: 500$ in blocking buffer, followed by $4 \times 5$ min washes in $0.9 \%$ $\mathrm{NaCl}-0.1 \%$ Tween-20 and $1 \mathrm{~h}$ incubation with ExtrAvidin- alkaline phosphatase (Sigma-Aldrich) at $1: 5000$. Another $4 \times 5$ min washing cycle was done and specific IgE visualization was performed by chromogenic reaction with nitroblue tetrazolium/5-bromo-4-chloro-3-indolyl phosphate (Sigma-Aldrich) in substrate buffer. Controls (B - blank) were performed with sera from 4 non-atopic dogs, negative for specific IgE to grass pollens.

\section{Statistical analysis}

Data distribution was verified with the KolmogorovSmirnov test and homogeneity of variance assessed by the Levene's test. As there was no normal distribution, the correlation between two variables was determined by the Spearman's rank correlation coefficient, considering $p<0.05$ as significant.

\section{Results}

All patients presented with specific IgE and positive IDT for D. glomerata and/or P. pratense. Mean specific IgE was 753.0 Elisa absorbance units (EAU) to D. glomerata (min. of 92 and max. of 3204) with a standard deviation (SD) of 738.4, and 874.8 EAU to P. pratense (min. of 89 and max. of 3426; SD = 894.0).

From D. glomerata IEF separation, at least 17 allergens were identified from pl 3.7 to 9.1 (Figure 1 and Table 1). From these 17, 10 bands (pl 3.9, 4, 4.2, 4.3, 4.4, 4.8, 5.4, 7.6, 7.7 and 9.1) showed major recognition (Table 1 and Figure 1). The mean number of D. glomerata recognized IEF allergen bands/patient was 9.2 (min. of 3 and max. of 15 ; SD = 3.38). Two allergen bands ( $p / 5.4$ and 7.6) were recognized by more than $85 \%$ of the patients and another one ( $\mathrm{pl} 4.8$ ) was recognized by all patients, while two others (pl 3.7 and 6.5) were only recognized by one patient, each (no. 9 and no. 8, respectively). From P. pratense IEF separation, at least 18 allergens were identified from pl 4 to 9.8. From these 18, seven bands showed major recognition ( $\mathrm{pl} 4$, 4.5, 4.8, 4.9, 5.4, 8.1 and 9.8), while three bands ( $\mathrm{pl} 7.3,7.6$ and 9.4) were only recognized by one patient (no. 3) and patient no. 9 only recognized two allergens ( $\mathrm{pl} 4$ and 6.1) (Table 2 and Figure 1). The mean number of $P$. pratense recognized IEF allergen bands/patient was 6.9 (min. of 2 and max. of 17; SD = 4.15).

From D. glomerata 1-D SDS-PAGE, 12 allergens were identified by patient sera, with molecular weights (MW) from 11 to $110 \mathrm{kDa}$. Seven of these allergen bands (MW close to $16,23,27,35,42,45$ and $65 \mathrm{kDa}$ ) showed major recognition. The mean number of $D$. glomerata recognized PAGE allergen bands/patient was 6.9 (min. of 4 and max. of 8; SD = 1.09). Six allergen bands $(16,27$, $35,42,45,65 \mathrm{kDa}$ ) were recognized by more than $85 \%$ of the patients and two bands (MW close to 43.5 and $110 \mathrm{kDa}$ ) were only recognized by one patient (no. 12). From P. pratense 1-D SDS-PAGE, eight allergen bands were recognized from 11 to $136 \mathrm{kDa}$ and the mean number of recognized PAGE allergen bands/patient was 4.4 (min. of 
Table 1. Allergens recognized from D. glomerata IEF

\begin{tabular}{|c|c|c|c|c|c|c|c|c|c|c|c|c|c|c|c|c|c|}
\hline \multirow[t]{2}{*}{ Patients } & \multicolumn{17}{|c|}{ Dactylis glomerata allergens (estimated $\mathrm{pl}^{\star}$ ) } \\
\hline & 3.7 & 3.9 & 4 & 4.2 & 4.3 & 4.4 & 4.8 & 5 & 5.4 & 5.7 & 6.5 & 6.8 & 7.6 & 7.7 & 8.3 & 8.7 & 9.1 \\
\hline 1 & & $x$ & $x$ & $x$ & $x$ & $\mathrm{x}$ & $x$ & $\mathrm{x}$ & $\mathrm{x}$ & & & & $\mathrm{x}$ & $\mathrm{x}$ & & & $x$ \\
\hline 2 & & $x$ & $\mathrm{x}$ & $x$ & & & $\mathrm{x}$ & & $\mathrm{x}$ & & & $\mathrm{x}$ & $\mathrm{x}$ & & & & \\
\hline 3 & & $x$ & $x$ & $x$ & $x$ & $x$ & $x$ & $x$ & $x$ & $x$ & & $x$ & $x$ & $x$ & $x$ & $x$ & $x$ \\
\hline 4 & & $x$ & $x$ & $x$ & $x$ & $x$ & $x$ & $x$ & $x$ & $x$ & & $x$ & $x$ & $x$ & $x$ & & $x$ \\
\hline 5 & & $x$ & $x$ & $x$ & & & $x$ & & $x$ & & & $x$ & $x$ & $x$ & & & \\
\hline $6 \mathrm{~B}$ & & $x$ & & $x$ & $x$ & $x$ & $x$ & & $x$ & & & & $x$ & $x$ & & $x$ & $x$ \\
\hline $6 \mathrm{~A}$ & & $x$ & & $x$ & $x$ & $x$ & $x$ & $x$ & $x$ & & & & $x$ & $x$ & & & $x$ \\
\hline 7 & & & $x$ & & & & $x$ & $x$ & $x$ & & & $x$ & $x$ & & & $x$ & $x$ \\
\hline 8 & & & $x$ & & & & $x$ & & $x$ & & $x$ & & & & & $x$ & $x$ \\
\hline 9 & $x$ & & & $x$ & & & $x$ & & & & & & $x$ & & & & \\
\hline 10 & & $x$ & & & & & $x$ & & & & & & $x$ & & & & \\
\hline 11 & & & $x$ & $x$ & $x$ & $x$ & $x$ & $x$ & $x$ & & & & $x$ & & & $x$ & \\
\hline 12 & & $x$ & $x$ & $x$ & $x$ & $x$ & $x$ & & $x$ & & & $x$ & & $x$ & $x$ & $x$ & $x$ \\
\hline 13 & & $x$ & $x$ & $x$ & & $x$ & $x$ & & $x$ & & & & $x$ & & $x$ & & $x$ \\
\hline 14 & & $x$ & $x$ & $x$ & $x$ & $x$ & $x$ & $x$ & $x$ & & & & $x$ & $x$ & $x$ & & $x$ \\
\hline
\end{tabular}

Table 2. Allergens recognized from $P$. pratense IEF

\begin{tabular}{|c|c|c|c|c|c|c|c|c|c|c|c|c|c|c|c|c|c|c|}
\hline \multirow[t]{2}{*}{ Patients } & \multicolumn{18}{|c|}{ Phleum pratense allergens (estimated $\mathrm{pl}^{\star}$ ) } \\
\hline & 4 & 4.5 & 4.6 & 4.8 & 4.9 & 5.1 & 5.4 & 5.6 & 6.1 & 7.1 & 7.3 & 7.6 & 8.1 & 8.8 & 9 & 9.4 & 9.5 & 9.8 \\
\hline 1 & & $x$ & $x$ & & $x$ & $x$ & $x$ & $x$ & $x$ & $x$ & & & $x$ & $x$ & $x$ & & & $x$ \\
\hline 2 & $x$ & $x$ & & & & & & & & & & & & $x$ & $x$ & & & \\
\hline 3 & $x$ & $x$ & & $x$ & $x$ & $x$ & $x$ & $x$ & $x$ & $x$ & $x$ & $x$ & $x$ & $x$ & $x$ & $x$ & $x$ & $x$ \\
\hline 4 & $x$ & $x$ & & & $x$ & & $x$ & & & & & & & & & & & $x$ \\
\hline 5 & & & & & $x$ & $x$ & $x$ & & & & & & & & $x$ & & & \\
\hline $6 \mathrm{~B}$ & $x$ & & & $x$ & & & & & & & & & & & & & $x$ & $x$ \\
\hline $6 \mathrm{~A}$ & $x$ & & & $x$ & $x$ & & $x$ & & & & & & & & & & & \\
\hline 7 & & $x$ & & $x$ & $x$ & & $x$ & & & & & & $x$ & $x$ & $x$ & & $x$ & $x$ \\
\hline 8 & & $x$ & & $x$ & $x$ & & $x$ & & & & & & $x$ & & & & & \\
\hline 9 & $x$ & & & & & & & & $x$ & & & & & & & & & \\
\hline 11 & & & & $x$ & $x$ & & $x$ & & & & & & $x$ & $x$ & & & $x$ & $x$ \\
\hline 13 & $x$ & $x$ & $x$ & & $x$ & & $x$ & & & $x$ & & & $x$ & $x$ & & & $x$ & $x$ \\
\hline 14 & $x$ & $x$ & & $x$ & $x$ & & $x$ & & & & & & $x$ & & & & & $x$ \\
\hline
\end{tabular}

1 and max. of $6 ;$ SD = 1.28). Three allergen bands (MW close to 27, 35 and $42 \mathrm{kDa}$ ) showed major recognition, with the $27 \mathrm{kDa}$ band being recognized by all the patients.

Patient no. 6, who had undergone 1-year ASIT, with a significant clinical improvement, changed his pattern of recognition towards the $D$. glomerata allergom (Tables 1 and 3), by failing to recognize the pl 8.7 (Figure 1) and the $47 \mathrm{kDa}$ (Figure 2) bands and beginning to recognize the $\mathrm{pl}$ 5 band (Figure 1). Patient no. 6 also changed his pattern of recognition regarding P. pratense (Tables 2 and 4), by failing to recognize the pl 9.5 and 9.8 (Figure 1) and the $11 \mathrm{kDa}$ (Figure 2) bands, and beginning to recognize the pl 4.9 and 5.4 bands (Figure 1). 
Table 3. Allergens recognized from $D$. glomerata SDSPAGE 1-D

\begin{tabular}{|c|c|c|c|c|c|c|c|c|c|c|c|c|}
\hline \multirow[t]{2}{*}{ Patients } & \multicolumn{12}{|c|}{ Dactylis glomerata allergens (estimated $\mathrm{MW}^{*}[\mathrm{kDa}]$ ) } \\
\hline & 11 & 16 & 23 & 27 & 32 & 35 & 42 & 43,5 & 45 & 47 & 65 & 110 \\
\hline 1 & & & $x$ & & $\mathrm{x}$ & $\mathrm{x}$ & & & & & $\mathrm{x}$ & \\
\hline 2 & & $\mathrm{x}$ & $x$ & $\mathrm{x}$ & & $\mathrm{x}$ & $x$ & & $\mathrm{x}$ & & $x$ & \\
\hline 3 & & $\mathrm{x}$ & $x$ & $\mathrm{x}$ & & $\mathrm{x}$ & $x$ & & $\mathrm{x}$ & $\mathrm{x}$ & $x$ & \\
\hline 4 & & $x$ & & $x$ & & $x$ & $x$ & & $x$ & & $x$ & \\
\hline 5 & & $x$ & & $x$ & & $x$ & $x$ & & $x$ & & $x$ & \\
\hline $6 \mathrm{~B}$ & & $x$ & $x$ & $x$ & & $x$ & $x$ & & $x$ & $x$ & $x$ & \\
\hline $6 \mathrm{~A}$ & & $x$ & $x$ & $x$ & & $x$ & $x$ & & $x$ & & $x$ & \\
\hline 7 & & $x$ & & $x$ & & $x$ & $x$ & & $x$ & $x$ & $x$ & \\
\hline 8 & & $x$ & & $x$ & & $x$ & $x$ & & $x$ & & $x$ & \\
\hline 9 & $x$ & $x$ & & $x$ & & $x$ & $x$ & & $x$ & & $x$ & \\
\hline 10 & $x$ & $x$ & & $x$ & & $x$ & $x$ & & $x$ & & $x$ & \\
\hline 11 & $x$ & $x$ & $x$ & $x$ & & $x$ & $x$ & & $x$ & & $x$ & \\
\hline 12 & & $x$ & $x$ & & $x$ & & & $x$ & $x$ & $x$ & $x$ & $x$ \\
\hline 13 & $x$ & $x$ & $x$ & $x$ & & $x$ & $x$ & & $x$ & & $x$ & \\
\hline 14 & & & $x$ & $x$ & & $x$ & $x$ & & $x$ & & $x$ & \\
\hline
\end{tabular}

*Molecular weight.

Two dimensional separation from D. glomerata disclosed 13 allergenic spots, from pl $<3.85$ to $>7.9$ and from 7 to $112 \mathrm{kDa}$ (Figure 3), while to P. pratense, 27 allergenic spots were disclosed from $\mathrm{pl}<3.85$ to $>9.7$ and from 9 to $85 \mathrm{kDa}$ (Figure 4).

Table 4. Allergens recognized from P. pratense SDS-PAGE 1-D

\begin{tabular}{|c|c|c|c|c|c|c|c|c|}
\hline \multirow[t]{2}{*}{ Patients } & \multicolumn{8}{|c|}{ Phleum pratense allergens (estimated $\mathrm{MW}^{*}[\mathrm{kDa}]$ ) } \\
\hline & 11 & 16 & 27 & 35 & 42 & 47 & 55 & 136 \\
\hline 1 & & & $x$ & $x$ & $x$ & & $x$ & \\
\hline 2 & $x$ & & $x$ & $\mathrm{x}$ & & & & $\mathrm{x}$ \\
\hline 3 & & & $x$ & $x$ & $x$ & & $x$ & $x$ \\
\hline 4 & & & $x$ & $\mathrm{x}$ & $x$ & & $x$ & $\mathrm{x}$ \\
\hline 5 & & & $x$ & $x$ & $\mathrm{x}$ & & & \\
\hline $6 \mathrm{~B}$ & $x$ & $x$ & $x$ & $x$ & $x$ & & & $x$ \\
\hline $6 \mathrm{~A}$ & & $x$ & $x$ & $x$ & $x$ & & & $x$ \\
\hline 7 & $x$ & & $x$ & $x$ & $x$ & & $x$ & \\
\hline 8 & $x$ & $x$ & $x$ & & & $x$ & & $x$ \\
\hline 9 & & & $x$ & & & & & \\
\hline 11 & & & $x$ & $x$ & $x$ & $x$ & $x$ & \\
\hline 13 & & & $x$ & $x$ & $x$ & & $x$ & \\
\hline 14 & & & $x$ & $x$ & $x$ & & $x$ & \\
\hline 15 & $x$ & & $x$ & $x$ & $x$ & $x$ & $x$ & \\
\hline
\end{tabular}

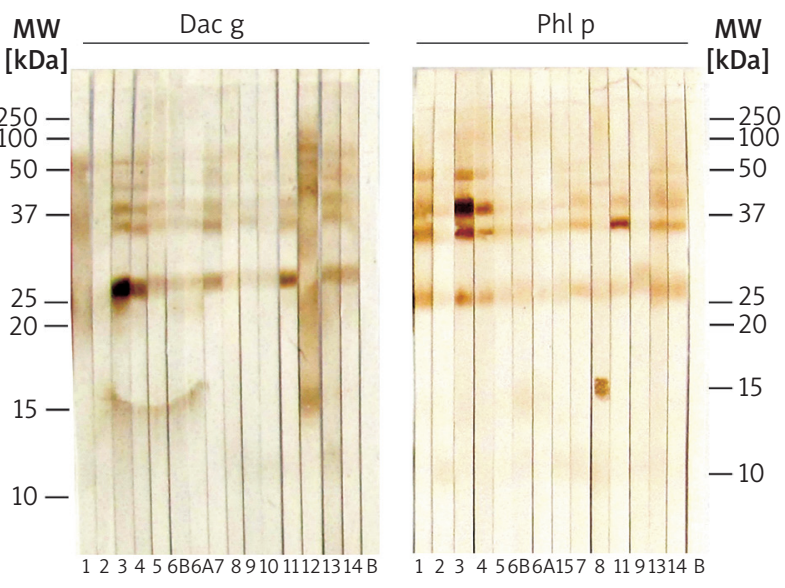

Figure 2. IgE immunoblots from D. glomerata and P. pratense SDS-PAGE, revealed with horseradish peroxidase-conjugated goat anti-dog-lgE. Patients/lanes no. 1-15. Patient no. 6 before (6B) and after (6A) specific immunotherapy. $B$ - Blank controls without serum. Note: no lane from patient no. 15 in the $D$. glomerata set and no lanes from patients no. 10 and 12 in the $P$. pratense set

In a range from 1 to 4 , the mean IDT score to $D$. glomerata was 2.3 (min. of 2 and max. of 4 ; SD = 0.602). To P. pratense, the mean IDT score was 2.0 (min. of 1 and max. of 3; SD = 0.516).

A significant positive correlation $(r=0.902)$ was verified between specific IgE to D. glomerata and the respective IDT scores, with a marked variability in individual specific IgE levels $(S D=738.4)$. A moderate positive correlation ( $r=0.422$ ) was found between specific IgE to $P$. pratense and the respective IDT scores, also with a marked variability in individual specific IgE levels (SD = 894.0). A significant positive correlation was verified between specific IgE, either to $D$. glomerata or to $P$. pratense, and their respective number of recognized IEF allergens $(r=0.570$ and $r=0.710)$. A significant positive correlation was also found between IDT scores to $D$. glomerata and to $P$. pratense, and to their respective number of recognized IEF allergens $(r=0.649$ and $r=0.660$ ). No significant correlation was verified between IDT scores to D. glomerata and to P. pratense, and their respective number of recognized PAGE allergens $(r=-0.070$ and $r=-0.216)$. No significant correlation was also verified between specific IgE to $D$. glomerata or to $P$. pratense and the number of their recognized PAGE allergens ( $r=0.009$ and $r=-0.036$, respectively).

A significant positive correlation was found between specific IgE levels to D. glomerata and P. pratense $(r=0.706)$, and between the number of $D$. glomerata and $P$. pratense recognized IEF allergens $(r=0.604)$. Between the number of $D$. glomerata and $P$. pratense recognized PAGE allergens, no significant correlation was observed $(r=0.152)$, whereas between IDT scores to both species, a moderate positive correlation was observed $(r=0.428)$. Besides, there was no significant correlation between the number 


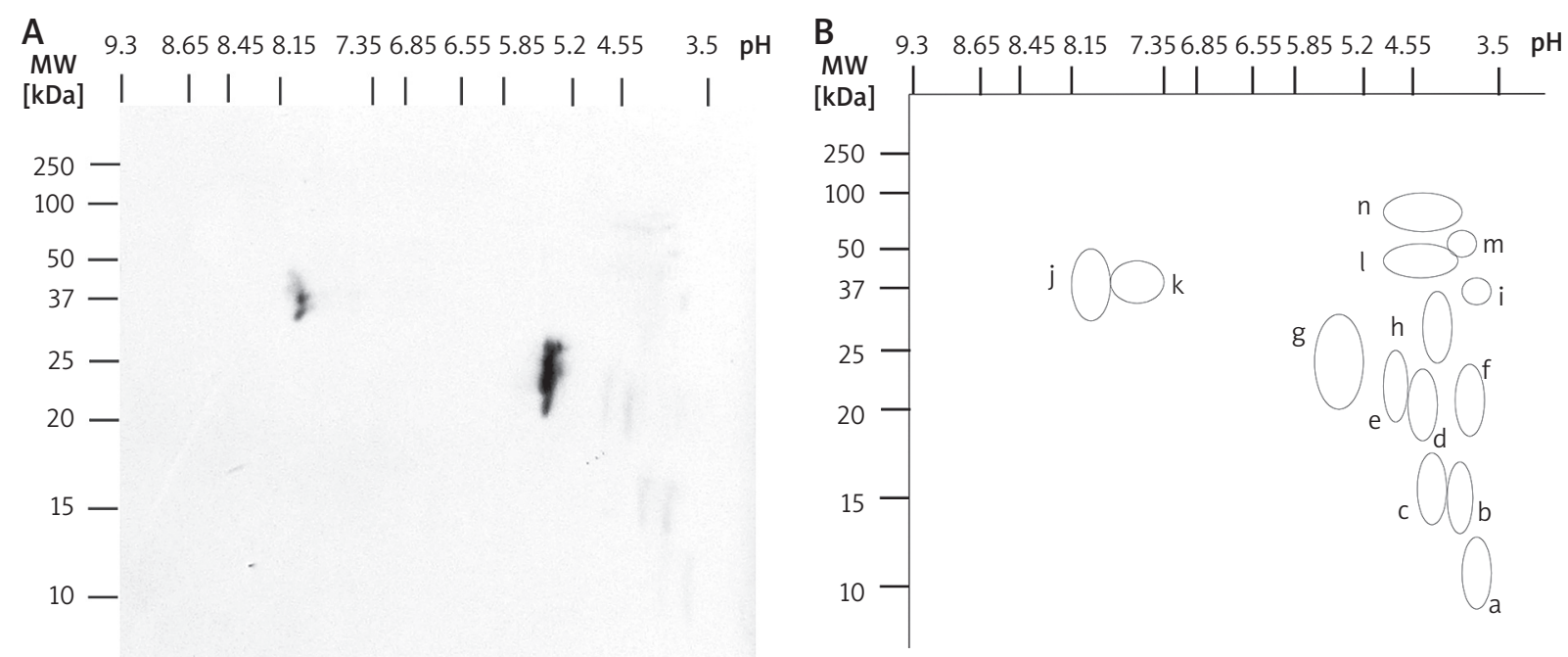

Figure 3. A - IgE immunoblot from D. glomerata 2-D separation, revealed with biotin-ExtrAvidin-alkaline phosphatase anti-dog-lgE. Dog D. glomerata allergogram with a pool of sera. B - Two-dimensional diagram from dog D. glomerata allergogram in Figure $3 \mathrm{~A}$. Recognized allergen spots are identified from $a$ to $n$

A

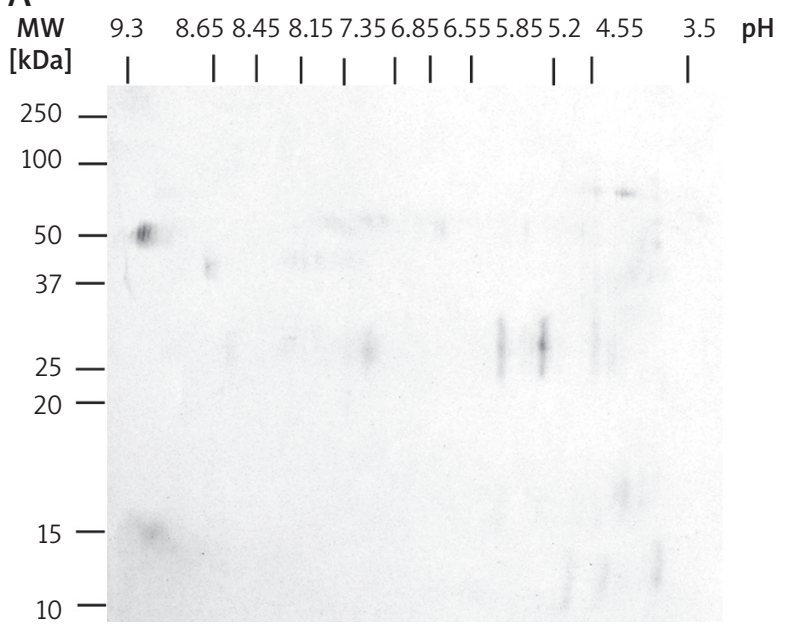

B

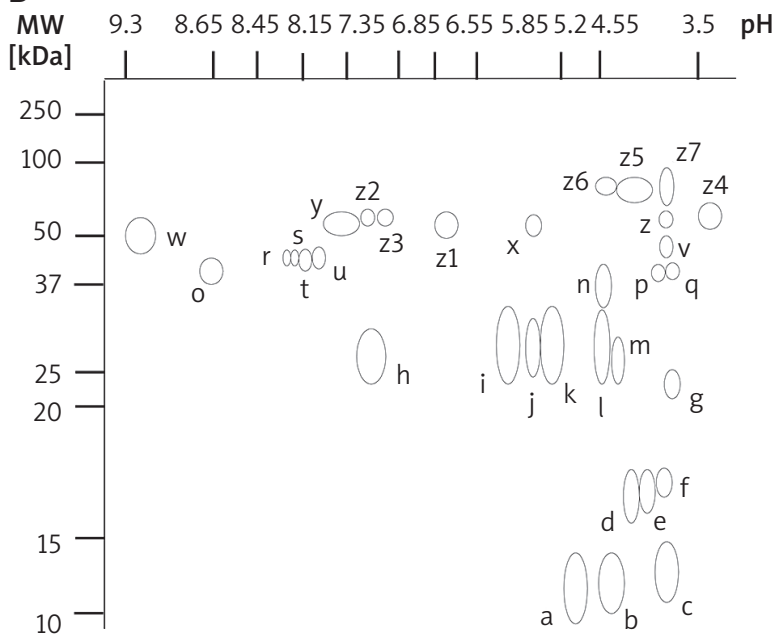

Figure 4. A - IgE immunoblot from P. pratense 2-D separation, revealed with biotin-ExtrAvidin-alkaline phosphatase antidog-lgE. Dog P. pratense allergogram with a pool of sera. B - Two-dimensional diagram from dog P. pratense allergogram in Figure 4 A. Recognized allergen spots are identified from $a$ to $z 7$

of recognized allergens from IEF and from PAGE, by each patient, in either D. glomerata or P. pratense. Although, a tendency to recognize a higher number of IEF allergens, when compared to PAGE allergens, was individually seen.

Two-dimensional separation disclosed multiple allergen spots from D. glomerata and P. pratense. Fourteen spots ( $a$ to $n$ ) were identified from $D$. glomerata ( $p$ l 3.8 to 7.9 and MW 7 to $75 \mathrm{kDa}$ ) (Figures $3 \mathrm{~A}$ and B) and 33 ( $a$ to $z 7$ ) from P. pratense ( $\mathrm{pl}<3.5$ to 9.1 and MW 8 to $83 \mathrm{kDa}$ ) (Figures $4 \mathrm{~A}$ and B). Diagrams from Figures $3 \mathrm{~B}$ and $4 \mathrm{~B}$ show clearer representations of $D$. glomerata and $P$. pratense 2-D allergoms. Controls with the non-atopic dogs, negative for specific lgE to grass pollens, showed no recognition in WB.

\section{Discussion}

No clear clinical differences were possible to identify among patients. Hence, no specific IgE level, IDT score or pattern of spectrotype was possible to relate to a specific clinical pattern. Similarly to house-dust mite allergy [24], IDT scoring seemed to be a reliable parameter in terms of correspondence to the level of specific IgE to D. glomerata and P. pratense. However, such high correlation may 
not occur between specific IgE levels or IDT scores and the number of recognized allergens from PAGE.

From the individual allergograms observed among this clinically homogeneous group of patients, sensitization profiles were found to be rather diverse. In fact, a great diversity of individual spectrotype patterns was revealed - patient no. 9 recognized only 1 allergen band from $P$. pratense PAGE, while others, such as patients no. 6 and 14, recognized up to 6 bands. From P. pratense IEF, patient no. 9 recognized only 2 bands, while patient no. 3 recognized 17. The same trend was registered with regard to $D$. glomerata $\mathrm{PAGE}$, where patient no. 1 recognized just 4 allergen bands, while patients no. 3, 6, 11, 12 and 13 recognized up to 8 bands. From D. glomerata IEF, the number of bands recognized ranged from 3 (patient no. 10) to 15 (patient no. 3). The number of recognized IEF-separated allergens was definitely lower in individual dogs than in humans, where more than 30 bands were individually recognized [25].

Dog patients with higher specific IgE levels also showed a higher allergen recognition signal in WB, as described for humans by Peltre et al. [18, 25], using the radioactive protocol for IgE detection.

As observed with humans [26], a wide range of identified D. glomerata and P. pratense allergens was disclosed for dogs and several similarities were also found between dog and human D. glomerata and P. pratense sensitization profiles. The $\mathrm{pl}$ range of the identified $D$. glomerata allergens for dogs ( $p$ l 3.7-9.1) was similar to the humans' ( $\mathrm{pl} 4.1-10.5)$ and the P. pratense pl range of identified allergens for dogs ( $p l$ 4-9.8) was even closer to the human's range ( $p \mid$ 4.2-9.5). Towards D. glomerata an allergen band with $\mathrm{pl}$ 5.9, probably Dac g 1, was the most recognized by humans [19], whereas in dogs the closest pl of a recognized allergen band was 5.7 and it was only recognized by 2 patients (no. 3 and 4). Although regarding P. pratense, 2 allergen bands ( $\mathrm{pl} 4.9$ and 5.4), which may correspond to Phl p 5 (pl 4.2-7.5), a highly recognized allergen by humans [19], also showed highly recognition by individual dogs (10 out of 12 dogs).

Some similarities were also found between dog and human sensitization profiles, regarding PAGE-separated D. glomerata and P. pratense allergens. The $27 \mathrm{kDa}$ and the $11 \mathrm{kDa} D$. glomerata allergens, probably corresponding, to Dac g 5 and Dac g 2, respectively, two major allergens for humans, were also identified by dogs, but only the $27 \mathrm{kDa}$ band presented major recognition by dogs (12 out of 14 dogs). The $27 \mathrm{kDa}$ and the $11 \mathrm{kDa}$. pratense allergen bands, probably corresponding to Phl p 5 and Phl p 2, respectively, were also recognized by dogs and the $27 \mathrm{kDa}$ band was even recognized by all of the sensitized dogs. Yet, regardless of the highly cross-reactive condition observed between different grass pollen species in humans that was not quite demonstrated among dogs. In fact, considerable differences were found between each patient Dactylis glomerata and Phleum pratense allergograms in the study population, as it was also reported with regard to Cryptomeria japonica [11] and Ambrosia artemisiifolia [12].

As in humans [26], mean specific IgE, either to D. glomerata or P. pratense, also presented a very high variability, with no correspondence to the severity of clinical signs. Nevertheless, a significant positive correlation was found between specific IgE and IDT scores, especially regarding $D$. glomerata, which may be understandable since the cutaneous reaction upon intradermal inoculation of the allergen extract seems to depend on the level of existent specific lgE. However, no correspondence was verified between IDT scores and the severity of the clinical signs. The relevant positive correlation verified between the level of specific IgE to D. glomerata and $P$. pratense, and the respective number of recognized IEF allergens, but not with regard to PAGE allergens, could be associated with a more conformational condition of humoral epitopes, which may be further preserved by the IEF, as a native method. Observing the correlations, early verified between IDT and specific IgE, and between specific IgE and the number of IEF-separated allergens, a significant positive correlation between the IDT scores to $D$. glomerata and the number of $D$. glomerata IEF-separated allergens was expected, by the same reasoning. Although, such conditions were not verified with respect to the number of PAGE allergens, either from D. glomerata or $P$. pratense, where no significant correlation was found with the respective levels of specific IgE and IDT scores. No relevant correlation was also found between the number of D. glomerata or P. pratense IEF and PAGEseparated allergens.

Individual spectrotypes, mainly 2-D allergograms, has already shown great utility for the identification of the implicated allergen molecules in human latex allergy, as demonstrated by Chardin and Peltre [2]. The interpretation of molecular allergen recognition may also be of great importance for an efficient immunotherapy approach, since the use of rather specific molecular allergens, instead of traditional commercial extracts, resulted in an additional 60\% improvement following immunotherapy in humans [13], and it is honest to predict the same trend in dog. Therefore, previous knowledge of the individual sensitization allergograms should play a key role for more efficient allergen-directed immunotherapy in dog. Improvement in immunotherapy effectiveness may also be expected in dogs by the use of individualspecific vaccines with molecular allergens.

The usefulness of molecular epidemiology had also been defined by Bessot and Pauli [6], concerning mite allergy in humans, because it would lead to a better understanding of the cross-reaction phenomena between different mite species, either from the same family or not. Further knowledge on this subject will probably open new prospects for a more specific immunotherapy, based on genetically hypoallergenic variants of major allergens [6]. 
In our study, after a 1-year course of specific immunotherapy, patient no. 6 showed a remarkable clinical improvement, with a change in D. glomerata and P. pratense spectrotype complexity, which could be associated with several Th2-like immunological mechanisms, occurred in the initial phase of immunotherapy [27-29], and suggesting a lack of absolute correlation between sensitization and allergy. Such observations were also made by Martins et al. [24], with an allergic patient, regarding Dermatophagoides pteronyssinus mite spectrotypes, but so far it has not been possible to detect any correlation between that and the efficacy of immunotherapy. More patients should be studied to confirm any possible early/late evolution in sensitization during the course of specific immunotherapy. However, until a more "tailor-made" immunotherapy becomes available, based on a true component-resolved molecular diagnosis [15, 16], extract mixes for immunotherapy are certainly useful, because species such as grass pollens are widely spread throughout the world, and thus, those mixtures undoubtedly tend to reproduce the natural exposure environment, frequently with a beneficial clinical effect [9]. Nevertheless, immunotherapy may be safely improved by using more accurate allergen pools for specific immunotherapy, which is already being studied for human allergy management [6-10].

As a future trend we think that the concept of CRD [15] and consequent CRIT [16] may also be applied to veterinary allergology. A possible example of those concepts could be applied towards the rather dog-recognized band of $27 \mathrm{kDa}$, either from D. glomerata or P. pratense, which may correspond to group 5 of grass pollen allergens. Hence, future experiments should investigate the precise molecular weight mass and amino-acid sequence of those molecules, by matrix-assisted laser desorption/ ionization time-of-flight/time-of-flight (MALDI-TOF/TOF) Mass spectrometry (MS), as demonstrated by Leung and Pitts [30]. Additionally, the use of rather specific identifying antibodies in WB or WB inhibition, by previous incubation of patient sera with selected natural or recombinant allergen molecules, may also allow direct or indirect 2D molecular spot identification. In fact, CRD is already being applied to human allergy management, through the Immune Solid-phase Allergen Chip (ISAC - Thermo Fisher Scientific, USA) diagnostic resource. However, while this trademark method is still not available for veterinary purposes, we consider that the identification and characterization of the most relevant allergens, especially for dogs, should continue through other available methods. For a better characterization of these identified allergens, further studies using several allergen-specific antibodies, recombinant allergens and mass spectrometry techniques are presently in progress. Additionally, as proposed by Canonica et al. [31] in a consensus document for the World Allergy Organization, the microchip-based molecular epidemiology concept diagnosis will facilitate identify- ing (i) genuine versus cross-reactive sensitization in polysensitized patients, thereby improving the understanding of triggering allergens; (ii) the risk of severe systemic versus mild local reactions in food allergy, without the need for challenge testing with unnecessary consequences and (iii) patients and triggering allergens for immunotherapy. In fact, etiological diagnosis of pollen sensitization is usually difficult, because of a wide variety of sensitizing pollens, which is also associated with food allergy, frequently configuring a poly-sensitization status, due to several panallergens [32]. Associated with this polysensitization status in humans, a low concordance between skin-prick-tests (SPT) was observed, especially with regard to patients sensitized to panallergens, such as profilin and polcalcin. In these cases, patients presented many more positive results in SPT. Additionally, patients sensitized to profilin or to lipid-transfer proteins have been clearly associated with food allergy [32]. This etiopathogenic relation between food allergy and atopy also needs clarification for dogs and other animals.

Evidence of an allergen epitope community within the Pooideae subfamily of grass pollens was also found for dogs, since $P$. pratense itself showed a large variety of allergens in the 2-D allergom spectrum, standing in agreement with Martínez-Cócera et al. [10] and Hejl et al. [9] observations.

Dog, as well as horse and cat allergy framework, harbor similar important parameters, such as IgE, IgE receptor repertoire and expression pattern, and the same involved cell types (mast cells, eosinophils and T-regulatory cells) for sensitization and for allergy diagnosis, suggesting to be possible models for translational studies [33].

As for humans, studies of molecular epidemiology in dog allergy will allow clarifying the evolution of allergic response during immunotherapy. Further characterization of D. glomerata and P. pratense identified allergens, using allergen-specific antibodies, as well as recombinant allergens and mass spectrometry techniques is in preparation. Hence, besides the very close clinical patterns between different patients, the individual pattern of sensitization for each allergenic source may also become of an outstanding relevance to the success of a real allergen-specific immunotherapy.

In conclusion, no clear clinical differences were possible to identify from among patients. Hence, no specific IgE level, IDT score or pattern of sensitization was possible to relate to a specific clinical pattern. Specific IgE to D. glomerata and to P. pratense presented high variability, with no correspondence to the severity of clinical signs. Sensitization profiles were found to be rather diverse from patient to patient, with a lack of absolute correlation between sensitization and allergy. However, patients with higher specific IgE levels tended to show a higher allergen recognition signal in WB. Several similarities were found in the patient pattern of sensitization between 
D. glomerata and P. pratense, suggesting cross-reactive phenomena. Similarities were found between dog and human D. glomerata and $P$. pratense sensitization profiles. No correlation was found between specific IgE levels or IDT scores and the number of recognized allergens from PAGE. The IDT scoring seemed to be a reliable parameter in terms of correspondence to the level of specific IgE to D. glomerata and P. pratense. Characterization of a patient IgE sensitization profile by WB over electrophoretic-separated allergens may provide relevant information, especially to understand particular conditions related to cross-reaction phenomena and immunotherapy effectiveness. Further molecular epidemiology approach is needed to understand the role of the sensitization pattern in allergen-specific immunotherapy effectiveness in grass-pollen allergic dogs.

\section{Acknowledgments}

The authors thank BIAL Aristegui (Bilbao, Spain) for kindly providing some of the intradermal tests; Companhia Portuguesa Consumer Health (CPCH) (Alcabideche, Portugal) for kindly providing several dermatological medications and to UNIVET laboratories (Barcelona, Spain) for supporting some of the specific IgE determinations.

The authors additionally thank Ana Martins for proofreading the text.

The work was funded by: 1) FEDER Funds through the Operational Program for Competitiveness Factors COMPETE and National Funds through FCT - Foundation for Science and Technology under the Strategic Project PEst-C/AGR/UI0115/2011. 2) SFRH/BPD/48164/2008 and UE/ICAAM/BII/03/2009, Fundação para a Cięncia e Tecnologia, Fundo Europeu de Desenvolvimento Regional, Quadro de Referęncia Estratégico Nacional. The sponsors were not involved in the study design, collection, analysis and interpretation of data, in the writing of the manuscript or in the decision to submit it for publication.

\section{Conflict of interest}

The authors declare no conflict of interest.

\section{References}

1. Olivry T. International Task Force of Canine Atopic Dermatitis. New diagnostic criteria for canine atopic dermatitis. Vet Dermatol 2010; 21: 123-6.

2. Chardin H, Peltre G. Allergome: the characterization of allergens based on a 2D gel electrophoresis approach. Expert Rev Proteomics 2005; 2: 757-65.

3. Olivry T, DeBoer DJ, Favrot C, et al. Treatment of canine atopic dermatitis: 2010 clinical practice guidelines from the International Task Force on Canine Atopic Dermatitis. Vet Dermatol 2010; 21: 233-48.

4. MacDonald JM. Allergen specific immunotherapy for atopy. In: Proceedings of North American Veterinary Conference. 2009. http://www.ivis.org/proceedings/NAVC/2006/ SAE/139.asp?LA=1. Accessed 11 Jun 2011.
5. Willemse T, Bardagi M, Carlotti DN, et al. Dermatophagoides farinae-specific immunotherapy in atopic dogs with hypersensitivity to multiple allergens: a randomised, double blind, placebo-controlled study. Vet J 2009; 180: 337-42.

6. Bessot JC, Pauli G. Mite allergens: an overview. Eur Ann Allergy Clin Immunol 2011; 43: 141-56.

7. Asturias JA, Ibarrola I, Arilla MC, et al. Engineering of major house dust mite allergens Der $\mathrm{p} 1$ and Der $\mathrm{p} 2$ for allergenspecific immunotherapy. Clin Exp Allergy 2009; 39: 1088-98.

8. Casquete-Román E, Rosado-Gil T, Postigo I, et al. Contribution of molecular diagnosis of allergy to the management of pediatric patients with allergy to pollen. J Investig Allergol Clin Immunol 2009; 19: 439-45.

9. Hejl C, Wurtzen PA, Kleine-Tebbe J, et al. Phleum pratense alone is sufficient for allergen-specific immunotherapy against allergy to Pooideae grass pollens. Clin Exp Allergy 2009; 39: 752-9.

10. Martínez-Cócera C, Sastre J, Cimarra M, et al. Immunotherapy with a Phleum pratense allergen extract induces an immune response to a grass-mix allergen extract. J Investig Allergol Clin Immunol 2010; 20: 13-9.

11. Kubota S, Miyaji K, Shimo Y, et al. IgE reactivity to a Cry j 3, an allergen of Japanese cedar (Cryptomeria japonica) pollen in dogs with canine atopic dermatitis. Vet Immunol Immunopathol 2012; 149: 132-5.

12. Ognjenovic J, Milcic-Matic N, Smiljanic K, et al. Immunoproteomic characterization of Ambrosia artemisiifolia pollen allergens in canine atopic dermatitis. Vet Immunol Immunopathol 2013; 155: 38-47.

13. Pauli G, Malling HJ. The current state of recombinant allergens for immunotherapy. Curr Opin Allergy Clin Immunol 2010; 10: 575-81.

14. Fall BI, Niessner R. Detection of known allergen-specific IgE antibodies by immunological methods. Methods Mol Biol 2009; 509: 107-22

15. Jahn-Schmid B, Harwanegg C, Hiller R, et al. Allergen microarray: comparison of microarray using recombinant allergens with conventional diagnostic methods to detect allergen-specific serum immunoglobulin E. Clin Exp Allergy 2003; 33: 1443-9.

16. Valenta R, Lidholm J, Niederberger V, et al. The recombinant allergen-based concept of component-resolved diagnostics and immunotherapy (CRD and CRIT). Clin Exp Allergy 1999; 29: 896-904.

17. Hamilton RG, Adkinson NF. In vitro assays for the diagnosis of IgE-mediated disorders. J Allergy Clin Immunol 2004; 114: 213-25.

18. Peltre G, Brodard V, David B. The grass pollen allergens repertoire or to how many grass pollen allergens are we sensitive? J Allergy Clin Immunol 1992; 4: 140-2.

19. Anderson K, Lidholm J. Characteristics and immunobiology of grass pollen allergens. Int Arch Allergy Immunol 2003; 130: 87-107.

20. Lee KW, Blankenship KD, McCurry ZM. Performance characteristics of a monoclonal antibody cocktail-based ELISA for detection of allergen-specific IgE in dogs and comparison with a high affinity IgE receptor-based ELISA. Vet Dermatol 2009; 20: 157-64.

21. Muller H, Schweizer B. Proteins. In: Biochemical Applications for UV/Vis Spectroscopy. Norwalk: Perkin-Elmer Corp 1993; III 1-32.

22. Martins LM, Peltre G, Faro C, Inácio F. The Helix aspersa (Brown Garden Snail) allergen repertoire. Int Arch Allergy Immunol 2005; 136: 7-15. 
23. Westermeier R. Semy-dry blotting of proteins. In: Electrophoresis in practice. Westermeir R (ed.). VHC, Weinheim 1993; 189-96.

24. Martins LM, Marques AG, Pereira LM, et al. House-dust mite allergy - mapping of Dermatophagoides pteronyssinus allergens for dogs by two-dimensional immunoblotting. Postep Dermatol Alergol 2015; 32: 73-81.

25. Peltre G, Lapeyre J, David B. Heterogeneity of grass pollen allergens (Dactylis glomerata) recognized by IgE antibodies in human patients sera by a new nitrocellulose immunoprint technique. Immunol Lett 1982; 5: 127-31.

26. Correia S, Martins L, Peres, MJ, et al. IgE and IgG4 specific antibodies to Dactylis glomerata allergens. J Allergy Clin Immunol 1996; 97: 295.

27. Kindt TJ. Hypersensitivity reactions. In: Kuby - Immunology. Kindt TJ, Goldsby RA, Osborne BA (eds). WH Freeman \& Co, New York 2007; 371-400.

28. Jutel M, Akdis M, Budak F, et al. IL-10 and TGF-beta cooperate in the regulatory $T$ cell response to mucosal allergens in normal immunity and specific immunotherapy. Eur J Immunol 2003; 33: 1205-14.

29. Creticos PS, Van Metre TE, Mardiney MR, et al. Dose response of IgE and IgG antibodies during ragweed immunotherapy. J Allergy Clin Immunol 1984; 73: 94-104.

30. Leung SM, Pitts RL. A novel approach using MALDI-TOF/ TOF mass spectrometry and prestructured sample supports (AnchorChip Technology) for proteomic profiling and protein identification. Methods Mol Biol 2008; 441: 57-70.

31. Canonica GW, Ansotegui IJ, Pawankar R, et al.; WAO-ARIAGA2LEN Task Force: Allen K, Asero R, Bohle B, Cox L, de Blay F, Ebisawa M, Maximiliano-Gomez R, Gonzalez-Diaz S, Haahtela T, Holgate S, Jakob T, Larche M, Matricardi PM, Oppenheimer J, Poulsen LK, Renz HE, Rosario N, Rothenberg M, Sanchez-Borges M, Scala E, Valenta R. A WAO-ARIA-GA2LEN consensus document on molecular-based allergy diagnostics. World Allergy Organ J 2013; 6: 17.

32. Orovitg A, Guardia P, Barber D, et al. Enhanced diagnosis of pollen allergy using specific immunoglobulin E determination to detect major allergens and panallergens. J Investig Allergol Clin Immunol 2011; 21: 253-9.

33. Jensen-Jarolim E, Einhorn L, Herrmann I, et al. Pollen allergies in humans and their dogs, cats and horses: differences and similarities. Clin Transl Allergy 2015; 7: 15. 